\title{
PREDICTORS OF VARICEAL BLEEDING AFTER ESOPHAGEAL VARICES BAND LIGATION IN EGYPTIAN CIRRHOTIC PATIENTS
}

\author{
Dr. Sahar Goda Zaghloul, Dr. Hoda Abdelaziz El Hady, Dr. Hussein Mohamed Hussein, \\ Islam Abdelsabour Hassan \\ Internal Medicine Department, Faculty of Medicine - zagazig University
}

\begin{abstract}
Background:Bleeding from oesophageal varices is a severe complication of portal hypertension. Endoscopic variceal ligation (EVL) is the treatment of choice for acute variceal bleeding. It is also performed for primary and secondary prophylaxis of bleeding from oesophageal varices. After Endoscopic Band Ligation (EBL), patients are at risk of post-interventional bleeding; the risk factors for this complication are poorly evaluated.The aim of this work: was to evaluate the risk factors for predicting variceal bleeding after elective endoscopic variceal ligation (EVL). Patients and Methods: This study will be carried out in Zagazig University Hospital and El-Galaa Family Military Hospital. The patients was subjected to different demographic, clinical, biochemical, ultrasonographic and endoscopic findings. Results : The incidence of bleeding after elective EVL was 6\%. The results showed significant differences between the bleeders and non bleeders regards the severity of liver disease measured by Child-Pugh score, the platelets count, hemoglobin level, prothrombin time, the liver regards its ( size, echogenicity, irregularity of the surface, presence of HFL and Portosystemic collaterals ), the size and extension of varices. Conclusion: For prediction of variceal bleeding after elective EVL; We can rely on many characteristics, such as age, gender, liver function, severity of varices, number of rubber bands, and so forth. But as demonstrated by the multivariate analysis, there were only four independent risk factors among these, namely moderate to excessive ascites, PT > 18, number of rubber bands placed, size and extent of varices. These four risk factors may therefore be more meaningful than the others for predicting the occurrence of bleeding following elective EVL.
\end{abstract}

Corresponding author: Islam Abdelsabour Hassan/tel 01064107681

\section{INTRODUCTION}

$\mathbf{P}$ ortal hypertension is the main complication of cirrhosis that leads to both an increase in hepatic sinusoidal pressure and an increase in portal pressure gradient, that is, the pressure difference between the portal vein and the systemic veins ${ }^{[1]}$

Variceal bleeding occurs at a yearly rate of $5 \%-15 \%$ in cirrhotic patients. The most important predictor of bleeding is the size of varices, with the highest risk of first bleeding (15\% per year) occurring in patients with large varices. Other predictors of bleeding are decompensated cirrhosis (Child-Pugh B/C) and the endoscopic presence of red wale marks ${ }^{[2]}$.

Trials have demonstrated that endoscopic variceal ligation (EVL) is an effective method to prevent variceal bleeding [3]. However, early recurrent bleeding after EVL mainly due to early spontaneous slippage of rubber bands leaving the unhealed ulcer $^{[4]}$.

There are only a few studies reporting the possible predictors for bleeding after EVL. Furthermore, the emergency EVL is often supposed to be different from the elective one because of the different patient conditions and technical difficulty ${ }^{[4]}$.

The possible predictive factors for bleeding after EVL: previous upper variceal bleeding, peptic esophagitis, a high platelet ratio index score, coagulation function, and number of varices ${ }^{[5]}$. Until now, there has been no general consensus on the risk factors and measures to prevent variceal bleeding ${ }^{[4]}$.

\section{AIM OF WORK}

The aim of this work was to evaluate the risk factors for predicting variceal bleeding after elective endoscopic variceal ligation (EVL).

\section{PATIENTS AND METHODS}

This study will be carried out in Zagazig University Hospital and El-Galaa Family Military Hospital. The patients was subjected to different demographic, clinical, biochemical, ultrasonographic and endoscopic findings.

All patients were followed up for 6 weeks to evaluate the risk factors that predict bleeding after elective endoscopic band 
ligation and they were divided into two groups:

GROUP (I): patients with post banding variceal bleeding (bleeding group).

GROUP (II): patients without post banding variceal bleeding (non bleeding group).

Comparisons were done between both groups to study the risk factors that predict bleeding.

The study protocol was approved by the local ethics committee and an informed consent was given prior to the study from all patients.

\section{Inclusion criteria:}

- Fifty cirrhotic patients duo to viral hepatitis or other causes submitted to elective EVL at our endoscopy unite for treatment of varices due to cirrhosis.

\section{Exclusion criteria:}

- Patients previously diagnosed to have other causes of upper GIT bleeding (such as: peptic ulcer disease, reflux esophagitis, erosions, antral vascular ectasia).

- Patients who have done injection sclerotherapy after endoscopic band ligation.

- Patients with blood diseases (such as: leukaemias, haemophilias, lymphomas, thrombocytopenic purpura).

All patients were subjected to the following:

I- Complete History Taking with stress on:

- Age.

- Gender.

- Number of attacks of upper GIT bleeding.

- Number of band ligation.

- History suggesting infection: spontaneous bacterial peritonitis (SBP) and non SBP.

- History of bleeding tendency.

II- II- Thorough Clinical Examination:

- General examination.

- Abdominal examination with stress on: Organomegaly,Manifestations of portal hypertension,Manifestations of liver cell failure and Manifestations suggesting infection.

III- Laboratory Investigations: CBC , Fasting Blood Sugar, Liver function tests (ALT , AST , bilirubin (total and direct), Serum albumin, Prothrombin time and concentration), Screening for the etiology of chronic liver diseases (Viral markers HBS-Ag and $\mathrm{HCV}-\mathrm{Ab}$ ), Alpha fetoprotein (AFP) and Renal function (S. creatinine) .

IV-Abdominal Ultrasonography with Stress on: Liver as regard its size, surface, echopattern and hepatic focal lesion (HFL) , PV diameter, PVT , Size of the spleen, Splenic vein diameter, Portosystemic collaterals and ascites.

$V$ - Evaluation of the patients according to Child classification.

VI-Upper GIT endoscopy regards grade of esophageal varices, Size of esophageal varices, Extension (upper, middle or lower third of esophagus), Risky signs e.g. red color signs and cherry red spots, Gastric varices and Portal hypertensive gastropathy (mild and severe).

The used machines were Pentax EPM 3500 videoscope \& FUJINON- EG - 250 HR2.

EVL was done under conscious sedation with $1 \mathrm{~cm}$ Midazolam to all patients as elective method for primary or secondary prophylaxis to prevent variceal bleeding.

Follow-up

Following EVL, standard doses of proton pump inhibitors (PPIs) were administered for $2 \mathrm{wk}$ for most patients. Food intake was allowed $24 \mathrm{~h}$ after the procedure in cases of prophylactic EVL. Bleeding was defined as Hematemesis, and/or melena, occurring within 6 weeks after EVL.

\section{STATISTICAL ANALYSIS}

The results were tabulated and statistical analysis was done and the results were considered non significant at $\mathrm{P}$ value $>0.05$, significant at $\mathrm{P}$ value $<0.05$ and highly significant at $\mathrm{P}$ value $<0.01$ 
Statistical presentation and analysis of the present study was conducted, using the mean, standard deviation, student t-test,
Fisher's exact test, Chi-square and Mann-

Whitney by SPS

RESULTS

Table (1): Comparison between bleeding and non bleeding groups regards age and gender:

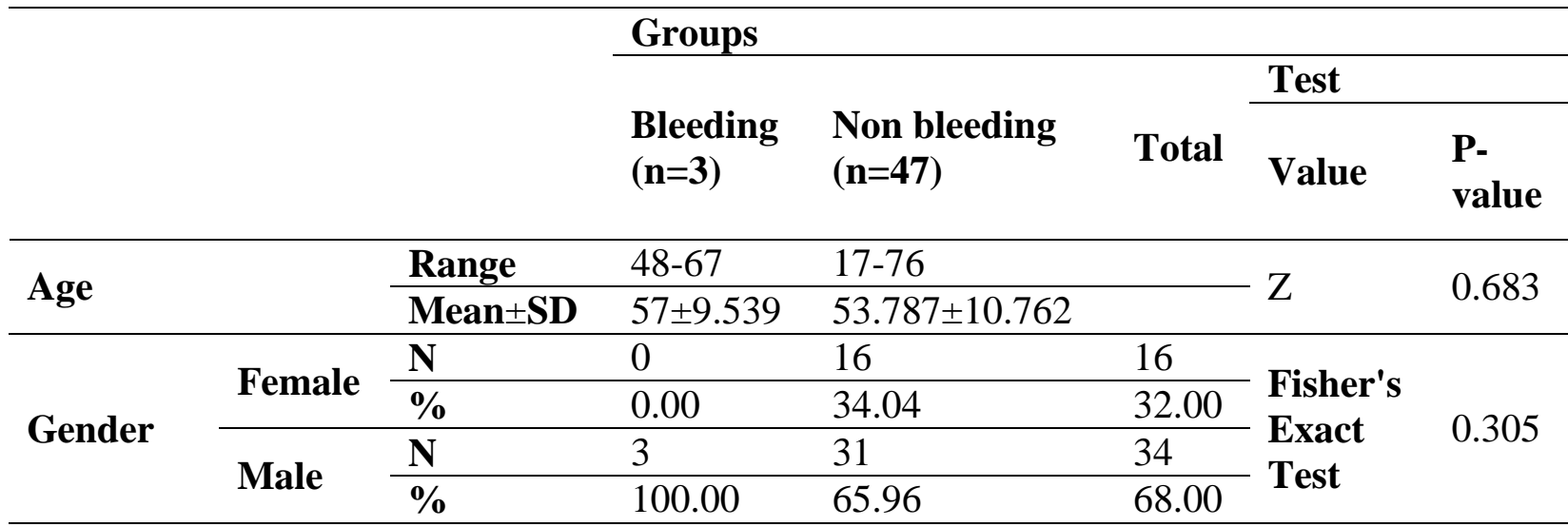

The above table shows that there was no statistically significant difference $(\mathrm{P}>0.05)$ between both groups regards age $\&$ gender.

Table (2): Comparison between bleeding and non bleeding groups regards habits and past history:

\begin{tabular}{|c|c|c|c|c|c|c|c|c|}
\hline \multirow{2}{*}{\multicolumn{2}{|c|}{$\begin{array}{l}\text { Habits and past } \\
\text { history }\end{array}$}} & \multicolumn{2}{|c|}{$\begin{array}{l}\text { Bleeding } \\
(\mathrm{n}=3)\end{array}$} & \multicolumn{2}{|c|}{$\begin{array}{l}\text { Non bleeding } \\
(\mathrm{n}=47)\end{array}$} & \multicolumn{2}{|c|}{ Total } & \multirow{2}{*}{ P-value } \\
\hline & & $\mathrm{N}$ & $\%$ & $\mathrm{~N}$ & $\%$ & $\mathrm{~N}$ & $\%$ & \\
\hline \multicolumn{2}{|l|}{ Smoker } & 1 & 33.33 & 13 & 27.66 & 14 & 28.00 & 0.636 \\
\hline \multicolumn{2}{|c|}{ Hypertension } & 1 & 33.33 & 9 & 19.15 & 10 & 20.00 & 0.496 \\
\hline \multicolumn{2}{|c|}{ Diabetes } & 2 & 66.67 & 14 & 29.79 & 16 & 32.00 & 0.237 \\
\hline \multicolumn{2}{|c|}{ Cardiac Disease } & 0 & 0.00 & 4 & 8.51 & 4 & 8.00 & 0.774 \\
\hline \multicolumn{2}{|c|}{ Renal Disease } & 0 & 0.00 & 4 & 8.51 & 4 & 8.00 & 0.774 \\
\hline \multirow{2}{*}{$\begin{array}{l}\text { History } \\
\text { of } \\
\text { surgery }\end{array}$} & Absent & 2 & 66.67 & 25 & 53.19 & 27 & 54.00 & \multirow{2}{*}{0.561} \\
\hline & Present & 1 & 33.33 & 22 & 46.81 & 23 & 46.00 & \\
\hline
\end{tabular}

The above table shows that there was no statistically significant difference $(\boldsymbol{P}>\mathbf{0 . 0 5})$ between both groups as regard Smoking, hypertension, diabetes, cardiac disease, renal disease and history of surgery. 
Table (3): Comparison between bleeding and non bleeding groups regards the etiology of cirrhosis:

\begin{tabular}{lllll}
\hline \multirow{2}{*}{ Etiology of cirrhosis } & & Groups & & \\
\cline { 2 - 5 } & & Bleeding $(\mathrm{n}=3)$ & $\begin{array}{l}\text { Non bleeding } \\
(\mathrm{n}=47)\end{array}$ & Total \\
\hline \multirow{2}{*}{$\mathrm{HCV}$} & $\mathrm{N}$ & 3 & 40 & 43 \\
\cline { 2 - 5 } & $\%$ & 100.00 & 85.11 & 86.00 \\
\hline \multirow{2}{*}{$\mathrm{HBV}$} & $\mathrm{N}$ & 0 & 5 & 5 \\
\hline \multirow{2}{*}{ Autoimmune } & $\mathrm{N}$ & 0.00 & 10.64 & 10.00 \\
\hline \multirow{2}{*}{ WILSON } & $\%$ & 0 & 1 & 1 \\
\hline \multirow{2}{*}{ Total } & $\mathrm{N}$ & 0.00 & 2.13 & 2.00 \\
\hline \multirow{2}{*}{ Chi-square } & $\%$ & 0.00 & 1 & 2.00 \\
\hline & $\mathrm{N}$ & 3 & 47 & 50 \\
\hline
\end{tabular}

The above table shows that there was no statistically significant difference $(\boldsymbol{P}>\mathbf{0 . 0 5})$ between both groups as regard etiology of cirrhosis.

Table (4): Comparison between bleeding and non bleeding groups regards results of laboratory investigations:

\begin{tabular}{|c|c|c|c|c|c|c|c|c|}
\hline \multicolumn{2}{|c|}{ laboratory investigations } & \multicolumn{5}{|l|}{ Groups } & \multicolumn{2}{|c|}{$\begin{array}{l}\text { Mann-Whitney } \\
\text { Test }\end{array}$} \\
\hline & & \multicolumn{3}{|c|}{ Bleeding $(n=3)$} & \multicolumn{2}{|c|}{ Non bleeding $(n=47)$} & $\mathrm{Z}$ & P-value \\
\hline \multirow{2}{*}{ ALT U/L } & Range & 24.000 & - & 37.000 & 13.500 & 45.000 & \multirow{2}{*}{-0.020} & \multirow{2}{*}{0.984} \\
\hline & Mean \pm SD & 31.333 & \pm & 6.658 & 31.489 & $\pm \quad 6.833$ & & \\
\hline \multirow{2}{*}{ AST U/L } & Range & 31.000 & - & 40.000 & 26.500 & - $\quad 44.700$ & \multirow{2}{*}{-0.572} & \multirow{2}{*}{0.567} \\
\hline & Mean \pm SD & 36.333 & \pm & 4.726 & 35.545 & $\pm \quad 4.016$ & & \\
\hline \multirow{2}{*}{ Albumin $\mathrm{g} / \mathrm{d}$} & Range & 1.800 & - & 2.200 & 1.600 & $-\quad 2.800$ & \multirow{2}{*}{-0.103} & \multirow{2}{*}{0.918} \\
\hline & Mean \pm SD & 2.033 & \pm & 0.208 & 2.057 & $\pm \quad 0.241$ & & \\
\hline \multirow{2}{*}{ PT second } & Range & 18.000 & - & 20.000 & 11.100 & - $\quad 18.200$ & \multirow{2}{*}{-2.085} & \multirow{2}{*}{0.042} \\
\hline & Mean \pm SD & 18.333 & \pm & 0.884 & 14.428 & \pm 1.714 & & \\
\hline \multirow{2}{*}{$\begin{array}{l}\text { T. Bilirubin } \\
\mathrm{mg} / \mathrm{d}\end{array}$} & Range & 3.000 & - & 4.300 & 2.200 & $-\quad 5.100$ & \multirow{2}{*}{-0.327} & \multirow{2}{*}{0.743} \\
\hline & Mean \pm SD & 3.600 & \pm & 0.656 & 3.681 & \pm 0.632 & & \\
\hline \multirow{2}{*}{ AKP } & Range & 90.000 & - & 140.000 & 53.700 & 150.900 & \multirow{2}{*}{-0.388} & \multirow{2}{*}{0.698} \\
\hline & Mean \pm SD & 115.333 & \pm & 25.007 & 110.091 & $\pm \quad 20.952$ & & \\
\hline \multirow{2}{*}{$\mathrm{Hb} \mathrm{g} / \mathrm{d}$} & Mean \pm SD & 5.900 & - & 9.000 & 8.000 & 12.200 & \multirow{2}{*}{-2.207} & \multirow{2}{*}{0.027} \\
\hline & Range & 7.900 & \pm & 1.735 & 9.860 & \pm 0.986 & & \\
\hline \multirow{2}{*}{ Hct \% } & Mean \pm SD & 17.000 & - & 27.900 & 13.000 & 36.000 & \multirow{2}{*}{-0.020} & \multirow{2}{*}{0.984} \\
\hline & Range & 22.300 & \pm & 5.456 & 22.536 & $\pm \quad 5.562$ & & \\
\hline
\end{tabular}




\begin{tabular}{|c|c|c|c|c|c|c|c|c|c|}
\hline \multirow{2}{*}{$\begin{array}{l}\text { WBC } \\
\text { thous/cmm }\end{array}$} & Mean \pm SD & 3.600 & - & 7.000 & 3.000 & - & 11.300 & \multirow{2}{*}{-1.696} & \multirow{2}{*}{0.090} \\
\hline & Range & 4.867 & \pm & 1.858 & 6.983 & \pm & 1.762 & & \\
\hline \multirow{2}{*}{$\begin{array}{l}\text { PLT } \\
\text { thous/cmm }\end{array}$} & Mean \pm SD & 60.000 & - & 88.000 & 43.000 & - & 124.000 & \multirow{2}{*}{-1.922} & \multirow{2}{*}{0.047} \\
\hline & Range & 72.667 & \pm & 14.189 & 93.574 & \pm & 17.809 & & \\
\hline \multirow{2}{*}{ ESR } & Mean \pm SD & 40.000 & - & 65.000 & 29.000 & - & 70.000 & \multirow{2}{*}{-0.184} & \multirow{2}{*}{0.854} \\
\hline & Range & 51.667 & \pm & 12.583 & 49.617 & \pm & 8.085 & & \\
\hline \multirow{2}{*}{ FBG mg/dl } & Mean \pm SD & 90.000 & - & 223.000 & 62.000 & - & 268.000 & \multirow{2}{*}{-0.225} & \multirow{2}{*}{0.822} \\
\hline & Range & 171.000 & \pm & 71.084 & 166.255 & \pm & 52.678 & & \\
\hline \multirow{2}{*}{$\begin{array}{l}\text { S.Creatinine } \\
\mathrm{mg} / \mathrm{dl}\end{array}$} & Mean \pm SD & 0.900 & - & 1.300 & 0.900 & - & 1.300 & \multirow{2}{*}{-0.173} & \multirow{2}{*}{0.863} \\
\hline & Range & & & & & & & & \\
\hline
\end{tabular}

The above table shows that there was no statistically significant difference $(P>0.05)$ between both groups as regarding ALT, AST, Albumin, total. Bilirubin, AKP, Hct, WBCs, ESR, S.creatinie and Fasting Blood Sugar (FBS), but there was statistically significant difference between both groups regarding prothrombin time $(\mathrm{PT})$, Hb level, Platelet count $(P<0.05)$.

Table (5): Comparison between bleeding and non bleeding regards (AFP):

\begin{tabular}{lllllll}
\hline \multirow{2}{*}{ AFP } & \multicolumn{2}{l}{$\begin{array}{l}\text { Bleeding } \\
(\mathrm{n}=3)\end{array}$} & \multicolumn{2}{l}{$\begin{array}{l}\text { Non bleeding } \\
(\mathrm{n}=47)\end{array}$} & Total \\
\cline { 2 - 8 } & $\mathrm{N}$ & $\%$ & $\mathrm{~N}$ & $\%$ & $\mathrm{~N}$ & $\%$ \\
\hline$<200 \mathrm{ng} / \mathrm{ml}$ & 1 & 33.33 & 43 & 91.49 & 44 & 88.00 \\
\hline$>200 \mathrm{ng} / \mathrm{ml}$ & 2 & 66.67 & 4 & 8.51 & 6 & 12.00 \\
\hline Total & 3 & 100.00 & 47 & 100.00 & 50 & 100.00 \\
\hline
\end{tabular}

The above table shows that there was statistically significant difference between both groups regarding (AFP) $(\boldsymbol{P}<\mathbf{0 . 0 5})$.

Table (6): Comparison between bleeding and non bleeding regards Child-Pugh classification:

\begin{tabular}{lllll}
\hline \multirow{2}{*}{ Child-Pugh classification } & & \multicolumn{2}{l}{ Groups } & \\
\cline { 2 - 5 } & & $\begin{array}{l}\text { Bleeding } \\
(\mathrm{n}=3)\end{array}$ & $\begin{array}{l}\text { Non bleeding } \\
(\mathrm{n}=47)\end{array}$ & Total \\
\hline \multirow{2}{*}{$\mathrm{A}$} & $\mathrm{N}$ & 0 & 12 & 12 \\
\cline { 2 - 5 } & $\%$ & 0.00 & 25.53 & 24.00 \\
\hline \multirow{2}{*}{$\mathrm{B}$} & $\mathrm{N}$ & 0 & 23 & 23 \\
\cline { 2 - 5 } $\mathrm{C}$ & $\%$ & 0.00 & 48.94 & 46.00 \\
\hline \multirow{2}{*}{ Total } & $\mathrm{N}$ & 3 & 12 & 30.00 \\
\hline \multirow{2}{*}{ Chi-square } & $\%$ & 100.00 & 25.53 & 50 \\
\hline
\end{tabular}

The above table shows that there was statistically significant difference between both groups regarding Child-Pugh classification $(\boldsymbol{P}<\mathbf{0 . 0 5})$. The bleeding patients had worse Child-Pugh scores, alls were class $\mathrm{C}$. On the other hand, $25.53 \%$ of non-bleeding were class A, $48.94 \%$ were class B, and $25.53 \%$ were class $\mathrm{C}(\mathrm{P}$ value $=0.021)$. 
Table (7): Comparison between bleeding and non bleeding regards infections:

\begin{tabular}{|c|c|c|c|c|c|c|c|c|}
\hline \multirow{2}{*}{ Infections } & & \multicolumn{2}{|c|}{$\begin{array}{l}\text { Bleeding } \\
(\mathrm{n}=3)\end{array}$} & \multicolumn{2}{|c|}{$\begin{array}{l}\text { Non bleeding } \\
(\mathrm{n}=47)\end{array}$} & \multicolumn{2}{|c|}{ Total } & \multirow{2}{*}{$\begin{array}{l}\mathrm{P}- \\
\text { value }\end{array}$} \\
\hline & & $\mathrm{N}$ & $\%$ & $\mathrm{~N}$ & $\%$ & $\mathrm{~N}$ & $\%$ & \\
\hline \multirow{2}{*}{$\begin{array}{l}\text { Ascitic Fluid } \\
\text { Infection (SBP) }\end{array}$} & Negative & 1 & 33.33 & 47 & 100.00 & 48 & 96.00 & \multirow{2}{*}{0.002} \\
\hline & Positive & 2 & 66.67 & 0 & 0.00 & 2 & 4.00 & \\
\hline \multirow{2}{*}{ Other Infections } & Negative & 3 & 100.00 & 39 & 82.98 & 42 & 84.00 & \multirow{2}{*}{0.586} \\
\hline & Positive & 0 & 0.00 & 8 & 17.02 & 8 & 16.00 & \\
\hline
\end{tabular}

The above table shows that there was statistically significant difference between bleeding and non-bleeding groups as regard infection with SBP $(\mathbf{P}$ value $=\mathbf{0 . 0 0 2})$ and it could be considered a risk factor for bleeding but there was no statistically significant difference between both groups as regard other infection $(\mathbf{P}$ value $\mathbf{= 0 . 5 8 6})$.

Table (8): Comparison between bleeding and non bleeding regards bleeding tendency:

\begin{tabular}{|c|c|c|c|c|}
\hline \multirow[b]{2}{*}{ Bleeding tendency } & & \multicolumn{3}{|l|}{ Groups } \\
\hline & & $\begin{array}{l}\text { Bleeding } \\
(n=3)\end{array}$ & $\begin{array}{l}\text { Non bleeding } \\
(\mathrm{n}=47)\end{array}$ & Total \\
\hline \multirow{2}{*}{ Present } & $\mathrm{N}$ & 0 & 11 & 11 \\
\hline & $\%$ & 0.00 & 23.40 & 22.00 \\
\hline \multirow{2}{*}{ Absent } & $\mathrm{N}$ & 3 & 36 & 39 \\
\hline & $\%$ & 100.00 & 76.60 & 78.00 \\
\hline \multirow{2}{*}{ Total } & $\mathrm{N}$ & 3 & 47 & 50 \\
\hline & $\%$ & 100.00 & 100.00 & 100.00 \\
\hline Fisher's Exact Test & & 0.466 & & \\
\hline
\end{tabular}

The above table shows that there was no statistically significant difference between both groups regarding bleeding tendency $(\boldsymbol{P}>\mathbf{0 . 0 5})$.

Table (9): Comparison between bleeding and non bleeding regards Ultrasonographic finding of the liver:

\begin{tabular}{|c|c|c|c|c|c|c|c|c|}
\hline \multicolumn{2}{|c|}{ Abd U/S of the Liver } & \multicolumn{2}{|c|}{$\begin{array}{l}\text { Bleeding } \\
(\mathrm{n}=3)\end{array}$} & \multicolumn{2}{|c|}{$\begin{array}{l}\text { Non bleeding } \\
(\mathrm{n}=47)\end{array}$} & \multicolumn{2}{|c|}{ Total } & \multirow{2}{*}{$\begin{array}{l}\mathrm{P}- \\
\text { value }\end{array}$} \\
\hline & & $\mathrm{N}$ & $\%$ & $\mathrm{~N}$ & $\%$ & $\mathrm{~N}$ & $\%$ & \\
\hline \multirow{3}{*}{ liver size } & Reduced & 2 & 66.67 & 5 & 10.64 & 7 & 14.00 & \multirow{3}{*}{0.043} \\
\hline & Average & 1 & 33.33 & 20 & 42.55 & 21 & 42.00 & \\
\hline & Enlarged & 0 & 0.00 & 22 & 46.81 & 22 & 44.00 & \\
\hline \multirow{3}{*}{ H.F.L } & NO & 1 & 33.33 & 43 & 91.49 & 44 & 88.00 & \multirow{3}{*}{0.017} \\
\hline & ONE & 1 & 33.33 & 4 & 8.51 & 5 & 10.00 & \\
\hline & Multiple & 1 & 33.33 & 0 & 0.00 & 1 & 2.00 & \\
\hline \multirow{2}{*}{ Liver surface } & Smooth & 0 & 0.00 & 31 & 65.96 & 31 & 62.00 & \multirow{2}{*}{0.049} \\
\hline & Irregular & 3 & 100.00 & 16 & 34.04 & 19 & 38.00 & \\
\hline \multirow{3}{*}{$\begin{array}{l}\text { Liver } \\
\text { echopattern }\end{array}$} & Coarse + & 0 & 0.00 & 10 & 21.28 & 10 & 20.00 & \multirow{3}{*}{0.024} \\
\hline & Coarse ++ & 0 & 0.00 & 25 & 53.19 & 25 & 50.00 & \\
\hline & Coarse +++ & 3 & 100.00 & 12 & 25.53 & 15 & 30.00 & \\
\hline
\end{tabular}

Ultrasonographic evaluation of the liver as regard its size, surface, echogenicity, and presence of HFL were all significantly different between both groups $(\boldsymbol{P}<\mathbf{0 . 0 5})$. 
Fig (1): Comparison between bleeding and non bleeding regards Ultrasonographic finding of the liver:

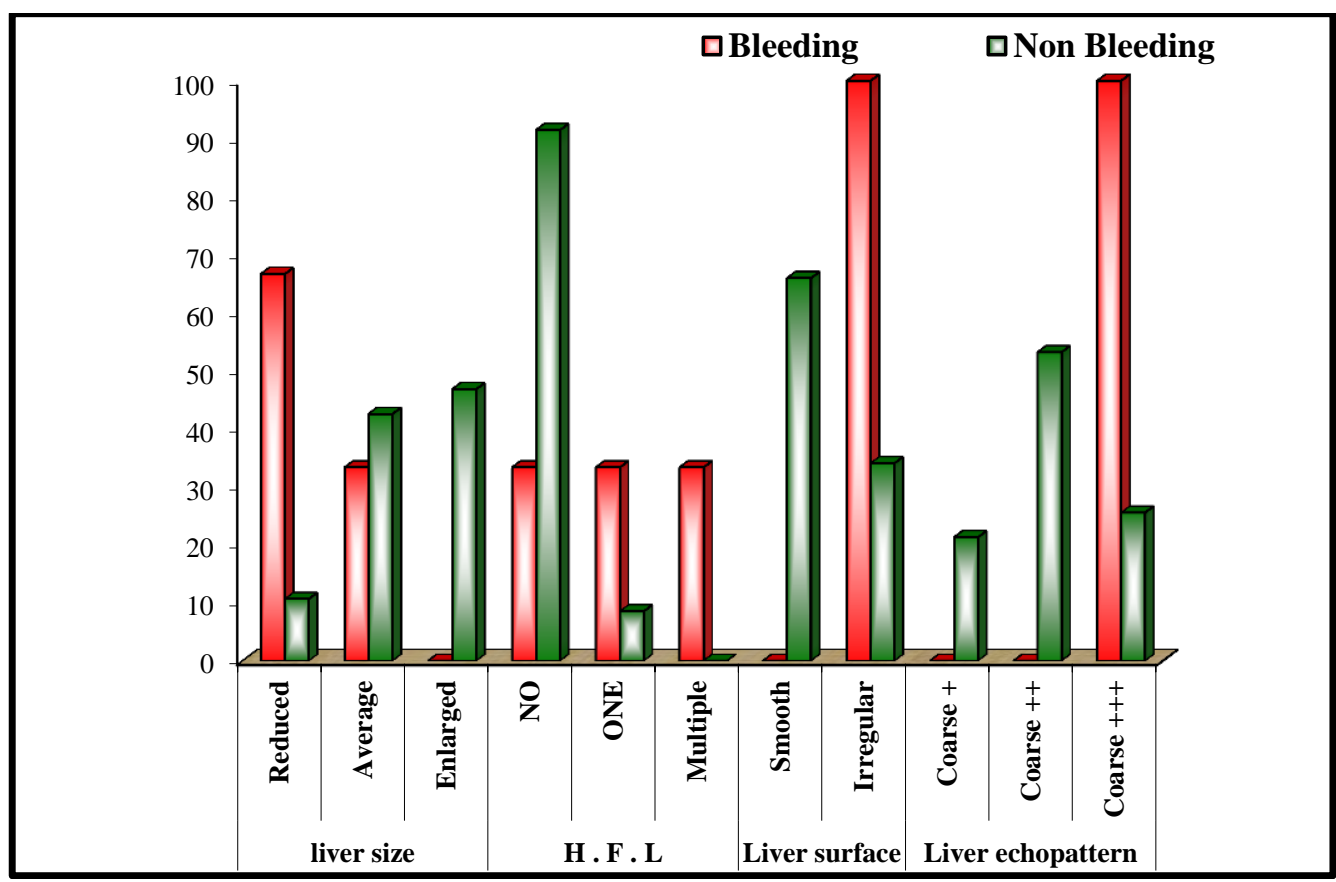

Table (10): Comparison between bleeding and non bleeding regards presence of portosystemic collaterals:

\begin{tabular}{lcccc}
\hline Abd U/S & \multicolumn{3}{c}{ Groups } \\
\hline portosystemic collaterals & & $\begin{array}{c}\text { Bleed } \\
\text { ing } \\
(\mathbf{n = 3})\end{array}$ & $\begin{array}{c}\text { Non } \\
\text { bleeding } \\
(\mathbf{n = 4 7 )}\end{array}$ & Total \\
\hline \multirow{2}{*}{ Present } & $\mathbf{N}$ & 2 & 2 & 4 \\
\cline { 2 - 5 } & $\mathbf{\%}$ & 66.67 & 4.26 & 8.00 \\
\hline \multirow{2}{*}{ Absent } & $\mathbf{N}$ & 1 & 45 & 46 \\
\hline & $\mathbf{\%}$ & 33.33 & 95.74 & 92.00 \\
\hline & $\mathbf{N}$ & 3 & 47 & 50 \\
\hline
\end{tabular}

Fisher's Exact Test

0.014

The above table shows that there was statistically significant difference between bleeding and non-bleeding groups as regard portosystemic collaterals $(\mathbf{P}$ value $=\mathbf{0 . 0 1 4})$ and it could be considered a risk factor for bleeding. 
Table (11): Comparison between bleeding and non bleeding regarding ascites:

\begin{tabular}{lllllll}
\hline \multirow{2}{*}{ Ascites } & \multicolumn{2}{l}{$\begin{array}{l}\text { Bleeding } \\
(\mathrm{n}=3)\end{array}$} & \multicolumn{2}{l}{$\begin{array}{l}\text { Non Bleeding } \\
(\mathrm{n}=47)\end{array}$} & Total & \multirow{2}{*}{ P-value } \\
\cline { 2 - 7 } & $\mathrm{N}$ & $\%$ & $\mathrm{~N}$ & $\%$ & $\mathrm{~N}$ & $\%$ \\
\hline No & 0 & 0.00 & 17 & 36.17 & 17 & 34.00 \\
\hline Mild & 0 & 0.00 & 13 & 27.66 & 13 & 26.00 \\
\hline Moderate & 1 & 33.33 & 10 & 21.28 & 11 & 22.00 \\
\hline Marked & 2 & 66.67 & 7 & 14.89 & 9 & 18.00 \\
\hline
\end{tabular}

The above table shows that there was statistically significant difference between bleeding and non-bleeding groups as regard ascites $(P$ value $=\mathbf{0 . 0 0 1})$ and it could be considered a risk factor for bleeding.

Table (12): Comparison between bleeding and non bleeding regards upper G.I. Endoscopic findings:

\begin{tabular}{|c|c|c|c|c|c|c|c|c|}
\hline \multirow[t]{2}{*}{ Upper G.I. } & \multirow[t]{2}{*}{ endoscopy } & \multicolumn{2}{|c|}{$\begin{array}{l}\text { Bleeding } \\
(\mathrm{n}=3)\end{array}$} & \multicolumn{2}{|c|}{$\begin{array}{l}\text { Non bleeding } \\
(\mathrm{n}=47)\end{array}$} & \multicolumn{2}{|c|}{ Total } & \multirow{2}{*}{ P-value } \\
\hline & & $\mathrm{N}$ & $\%$ & $\mathrm{~N}$ & $\%$ & $\mathrm{~N}$ & $\%$ & \\
\hline \multirow{5}{*}{$\begin{array}{l}\text { Grade of } \\
\text { varices }\end{array}$} & II & 0 & 0.00 & 3 & 6.38 & 3 & 6.00 & \multirow{5}{*}{0.453} \\
\hline & II - III & 0 & 0.00 & 10 & 21.28 & 10 & 20.00 & \\
\hline & III & 0 & 0.00 & 9 & 19.15 & 9 & 18.00 & \\
\hline & III-IV & 1 & 33.33 & 7 & 14.89 & 8 & 16.00 & \\
\hline & IV & 2 & 66.67 & 18 & 38.30 & 20 & 40.00 & \\
\hline \multicolumn{2}{|l|}{ Red sign } & 3 & 100.00 & 37 & 78.72 & 40 & 80.00 & 0.239 \\
\hline \multicolumn{2}{|c|}{ Gastric Varices } & 0 & 0.00 & 4 & 8.51 & 4 & 8.00 & 0.774 \\
\hline \multirow{4}{*}{ *PHG } & Negative & 0 & 0.00 & 18 & 38.30 & 18 & 36.00 & \multirow{4}{*}{0.131} \\
\hline & Mild & 2 & 66.67 & 14 & 29.79 & 16 & 32.00 & \\
\hline & Moderate & 0 & 0.00 & 11 & 23.40 & 11 & 22.00 & \\
\hline & Severe & 1 & 33.33 & 4 & 8.51 & 5 & 10.00 & \\
\hline
\end{tabular}

$* \boldsymbol{P H G}=$ portal hypertensive gastropathy

The above table shows that there was no statistically significant difference $(\boldsymbol{P}>$ 0.05) between both groups regarding grading of varices, red sign, presence of gastric varices and portal hypertensive gastropathy (PHG), however all patients in the bleeding group have esophageal varices grade III-IV \&grade IV, Also two of them have mild PHG \& One of them has sever PHG. So risk of bleeding increase in patients which having esophageal varices grade IV and sever PHG. 
Table (13): Comparison between bleeding and non bleeding regards medication after EVL:

\begin{tabular}{|c|c|c|c|c|c|c|c|c|}
\hline \multicolumn{2}{|c|}{ Medication after EVL } & \multicolumn{2}{|c|}{$\begin{array}{l}\text { Bleeding } \\
(n=3)\end{array}$} & \multicolumn{2}{|c|}{$\begin{array}{l}\text { Non bleeding } \\
(\mathrm{n}=47)\end{array}$} & \multicolumn{2}{|c|}{ Total } & \multirow{2}{*}{$\begin{array}{l}\mathrm{P}- \\
\text { value }\end{array}$} \\
\hline & & $\mathrm{N}$ & $\%$ & $\mathrm{~N}$ & $\%$ & $\mathrm{~N}$ & $\%$ & \\
\hline \multirow{2}{*}{$\begin{array}{l}\text { Post EVL } \\
\text { Sucralfate }\end{array}$} & Negative & 1 & 33.33 & 3 & 6.38 & 4 & 8.00 & \multirow{2}{*}{0.226} \\
\hline & Positive & 2 & 66.67 & 44 & 93.62 & 46 & 92.00 & \\
\hline \multirow{2}{*}{$\begin{array}{l}\text { Post EVL } \\
\text { Vit . K }\end{array}$} & Negative & 3 & 100.00 & 5 & 10.64 & 8 & 16.00 & \multirow{2}{*}{0.003} \\
\hline & Positive & 0 & 0.00 & 42 & 89.36 & 42 & 84.00 & \\
\hline \multirow{2}{*}{$\begin{array}{l}\text { Post EVL } \\
\text { B.Blocker }\end{array}$} & Negative & 3 & 100.00 & 35 & 74.47 & 38 & 76.00 & \multirow{2}{*}{0.430} \\
\hline & Positive & 0 & 0.00 & 12 & 25.53 & 12 & 24.00 & \\
\hline \multirow{2}{*}{$\begin{array}{l}\text { Post EVL Proton } \\
\text { pump inhibitor }\end{array}$} & Negative & 0 & 0.00 & 2 & 4.26 & 2 & 4.00 & \multirow{2}{*}{0.248} \\
\hline & Positive & 3 & 100.00 & 45 & 95.74 & 48 & 96.00 & \\
\hline
\end{tabular}

The above table shows that there was no statistically significant difference $(\boldsymbol{P}>\mathbf{0 . 0 5})$ between both groups regarding medication after EVL by Sucralfate, B.Blocker and Proton pump inhibitor.

There was statistically significant difference $(\boldsymbol{P}>\mathbf{0 . 0 5})$ between both groups regarding medication after endoscopic variceal ligation by Vit . K. All patients in bleeding groups not received Vit.K as medical treatment after EVL.So, it is recommended to give Vit.K to all patients after EVL.

Fig (2): Comparison between bleeding and non bleeding regards medication after EVL:

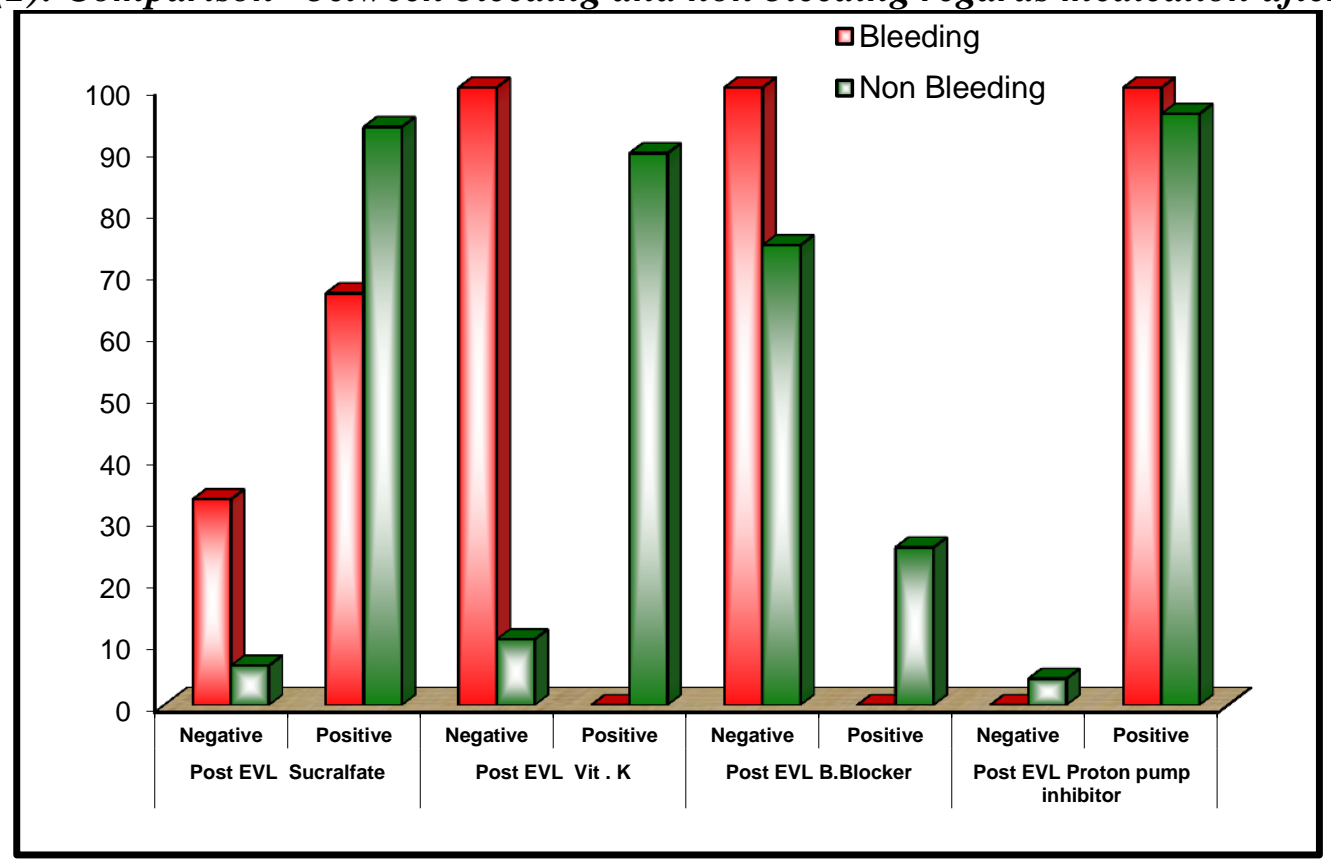

All patients in bleeding group were submitted to diagnostic upper endoscopy for detection the cause of bleeding post EVL. Sever PHG was the source of bleeding in the first case, post banding ulcers was the source of bleeding in the second case and large esophageal varices ( grade IV, bluish, beaded, risky with red color signs ) with Ls extension was the source of bleeding in the third case.

\section{DISCUSSION}

EVL is an effective method to prevent variceal bleeding primarily and secondarily. However, bleeding as a vital complication 
after elective EVL has not been studied fully. There are only a few studies reporting the possible predictors for bleeding after elective EVL $^{[4]}$.

[6] reported that the rate of early bleeding following EVL was between $9 \%$ and $19 \%$, which is close to our result, 3 patients $(6 \%)$. We also found that post-EVL bleeding was most likely to occur between the 7 th and 13th day following the procedure.

Age distribution in this study showed that patients with bleeding were slightly older than those who did not bleed mean age was $57 \pm 9.539$ years versus $53.7 \pm 10.762$ years respectively [7] reported similar results. Conflicting results were found as regard age by

Grothaus ${ }^{[1]}$ who reported that patients who bled were slightly younger than those who did not bleed. ${ }^{[8]}$ and ${ }^{[9]}$ reported that age was not a risk factor for bleeding. Nevertheless, the role of age as a risk factor needs further evaluation.

Male gender in this study was significant risk factor for bleeding. This contradicts other studies by Grothaus ${ }^{[1]}$ and Xu $\boldsymbol{e t} \boldsymbol{a l}^{[7]}$ who reported that gender was not significantly different between both groups. This conflict could be attributed to male predominance in our study population (all patients who bled were males).

As regard the previous history of frequent hematemesis attacks, we found that more attacks of hematemesis were not a significant risk for bleeding. Also we found that patients with history of more frequent EVL sessions were not protected against bleeding. This may be due to that the efficacy of eradication of varices after EVL depends on both the number of bands placed in each session and the time interval between sessions [10].

Infection with spontaneous bacterial peritonitis was a significant risk factor for bleeding. This finding was consistent with those of ${ }^{[1]} \&^{[7]} \&^{[11]}$

All patients with cirrhosis with ascites and variceal bleeding are at high risk of developing SBP because of their immunocompromised state, the disturbed function of the mucosal barrier, followed by increased bacterial translocation and the frequent invasive manipulations as part of diagnostic and therapeutic procedures [12]

Many studies reported a high incidence of bacterial infection in patients with bleeding events after EVL ranging from $14-66 \%\left({ }^{[8]},{ }^{[9]}\right.$ $\&{ }^{[1]}$ Our study reported an incidence of $66.67 \%$ in patients with bleeding, and $4 \%$ in non- bleeding patients.

Patients with decompensated cirrhosis often have coagulation disorders. The coagulation index as an independent predictive factor for bleeding after EVL was reported in some previous studies as ${ }^{[5]} \&^{[4]}$ but not in another as ${ }^{[13]}$. Our study showed that PT > 18 was an independent risk factor of post-EVL

bleeding

$($ Mean \pm SD18.333 \pm 0.884$) . \quad$ It is understandable that the ulcers caused by rubber bands can not heal well without normal coagulation. The prolongation of PT suggests a lack of coagulation factors I, II, VII or $\mathrm{X}$, or fibrinolysis acceleration. Therefore, for patients with quite prolonged PT, supplementing vitamin K1 and coagulation factors are necessary before EVL. However, we found that history suggesting bleeding tendency (in the form of epistaxis, bleeding per gums, ....) was not a significant risk factor for bleeding. This may be explained by the fact that bleeding tendency has a little relationship with abnormal clotting tests ${ }^{[14]}$.

Our study showed that the risk of bleeding was higher in patients with shrunken liver, more coarse hepatic echopattern, presence of irregular surface, and patients with HFL. We found that diameter of portal vein and splenic vein was not a risk factor for bleeding. This is not inagreement with prior studies which observed that diameter of portal vein and splenic vein was a risk factor for bleeding. So, monitoring of PV size offers an easy, available, non-invasive, reliable and cost effective way to evaluate cirrhotic patients for the risk of variceal bleeding ${ }^{[15]} \&$ ${ }^{[16]} \&^{[17]}$

Although ${ }^{[18]}$ reported that the presence of portosystemic collaterals was not an indicator of presence of large varices, we found that presence of collaterals is highly associated with bleeding. To the best of our knowledge, presence of collaterals has not 
been investigated regarding its predictive role in bleeding complications after EVL in comparable studies.

In this study, we found that the HB level and platelets count as possible risk factors for bleeding. We found that WBCs count was not significantly different between both groups. However, many authors as ${ }^{[8]} \&^{[5]} \&^{[1]}$ reported that WBCs count was significantly associated with bleeding and they correlated between leucocytosis and presence of infection. Our results may be explained by that leucopenia which is an indicator of presence of large varices may mask infection associated leukocytosis, this is in agreement with ${ }^{[18]} \&^{[19]}$. The main cause of leucopenia is $\mathrm{PH}$ and hypersplensim ${ }^{[20] .}$

On the other hand, there was a significant association between low hemoglobin level and bleeding. [1] \& [7] reported similar results. They found that patients with bleeding events after EVL had significantly lower hemoglobin levels. However, anemia may be considered a result rather than cause of bleeding, because most patients had multiple previous attacks of hematemesis and multiple EVL sessions. As regard the platelets count we found that thrombocytopenia was significantly different between both groups. These results were not in agreement with ${ }^{[21]} \&^{[1]} \&^{[7]}$.

In this study there was strong evidence between severity of liver disease, measured by Child-Pugh score, and bleeding. All bleeding cases had a Child score $\mathrm{C}$ and this was consistent with previously reported findings by ${ }^{[22]} \&^{[1]} \&{ }^{[7]}$ Our study showed that there was a difference in Child-Pugh score between the bleeding and non bleeding groups. Furthermore, we revealed that ascites and PT, two of the indices for Child-Pugh classification, were independent risk factors for bleeding after EVL, but the other three indices were not.

Ascites as an independent risk factor for bleeding after EVL was not reported in the study of ${ }^{[4]}$. However, they did not quantify the volume of ascites. We demonstrated that a moderate to excessive volume of ascites was the most dangerous factor predicting postEVL bleeding. This may be explained by the elevated portal vein pressure that results from a larger volume of ascites. It was reported in a previous study by ${ }^{[23]}$ that reported variceal bleeding recurred more in patients with higher basal portal vein pressure, and led to higher mortality.

In this study, we collected more expanded indices than former studies to evaluate patients with esophageal varices more comprehensively, which allowed us to draw convincing conclusions. For example, we took account of extent of varices, number of varices, number of rubber bands, portal vein diameter, PVT, and so on.

We also evaluated different endoscopic parameters in both groups. We found that $66.67 \%$ of bleeding patients had varices described as Ls, while only $6.38 \%$ of nonbleeding patients had Ls varices. As regard size of varices, we found that esophageal varices grade IV were present in $66.67 \%$ of bleeding patients A while it was present in $38.30 \%$ of non- bleeding patients. Red color sign was found in all bleeding patients. [7] reported similar results. He found that all the patients who bled had varices classified as "severe", while only $40 \%$ of the non- bleeders did. $\mathrm{He}$ also found that the percentage of patients with varices throughout the whole extent of the esophagus in the bleeding group (8 times) more than that of the non- bleeders, which is close to our result (10 times) more than that of the non- bleeders .

The presence of post EVL ulcer was a significant cause of bleeding in our study, This agreed with the results of ${ }^{[4]}$ who reported similar results, They found that cases of severe bleeding after EVL were all caused by early slippage of the rubber bands, leaving the unhealed ulcer. On the basis of the above result, recommending a soft diet and avoiding strenuous exercise is helpful in preventing early slippage of the rubber bands which may cause life threatening bleeding, On the other hand ${ }^{[23]}$ reported different results.

The results showed significant differences between the bleeders and non bleeders for many characteristics, such as age, gender, liver function, severity of varices, number of rubber bands, and so forth. But as demonstrated by the multivariate analysis, there were only four independent risk factors among these, namely moderate to excessive 
ascites, number of rubber bands placed, and extent of varices and PT $>18$. These four risk factors may therefore be more meaningful than the others for predicting the occurrence of bleeding following EVL.

${ }^{[24]}$ believed that the more rubber bands that were used to ligate, the greater the possibility of bleeding, because of the increasing ulcers.

In our study, we also found that the number of rubber bands was an independent risk factor for bleeding after EVL. Therefore, for varices which were in the mild to moderate class, it may not be reasonable to launch many rubber bands. For severe varices, however, it's usually unavoidable to use more bands.

We found the other independent risk factor was the extent of varices, which also reflects the severity of varices. Varices that extend along the entire esophagus are much more dangerous than varices that are limited to the middle and lower part.

On the other hand, a greater extent of varices often means that more rubber bands are needed, increasing the possibility of bleeding.

When considering the healing of postEVL ulcers, the use of PPIs has been reported useful in comparison with a placebo, but the effect on preventing bleeding was not conclusive. In this study, almost every patient received a standard dose of PPIs for $2 \mathrm{wks}$ after EVL, but there was no significant difference between the two groups. We also failed to find any benefit in the use of sucralfate for the prevention of bleeding related to post-banding ulcers. B-blocker is another useful drug to reduce portal vein pressure, and it can be taken for a long time. But the number of treated patients was very small and may not accurately reflect the facts.

\section{CONCLUSION}

\section{From our results we conclude that:}

- We cannot rely on number of attacks of hematemesis and number of previous EVL sessions for prediction of variceal bleeding after elective EVL.
- We can rely on severity of liver disease, measured by Child-Pugh score for prediction of variceal bleeding.

- As regarding the laboratory variables, we can rely on the platelets count, hemoglobin level, and prothrombin time for prediction of variceal bleeding and mortality.

- As regarding the ultrasonographic findings we can rely on the liver size, echogenicity, irregularity of the surface, presence of HFL and Portosystemic collaterals, but we can not rely on the Splenomegaly, PV patency, diameter of PV and splenic vein for prediction of variceal bleeding.

- As regarding the upper endoscopic variables, we can not rely on the grade of esophageal varices, red color signs, gastric varices and portal hypertensive gastropathy for prediction of variceal bleeding, but we can rely on the size and extension of varices for prediction of variceal bleeding.

\section{REFERENCES}

1. Grothaus J, Petrasch F, Zeynalova S, Mössner J, Schiefke I, Hoffmeister A Risk Factors for Bleeding Complications after Endoscopic Variceal Ligation Therapy. $Z$ Gastroenterol; (2010): 48:1200-1206.

2. Garcia-Tsao G., Sanyal A.J., Grace N.D., et al Prevention and management of gastroesophageal varices and variceal hemorrhage in cirrhosis. Hepatology; (2007): 46:922-938.

3. Khuroo M.S., Khuroo N.S., Farahat K.L.C., et al Meta-analysis: endoscopic variceal ligation for primary prophylaxis of oesophageal variceal bleeding. Alimentary pharmacology \& therapeutics; (2005): 21(4): 347-361.

4. Vanbiervliet G., Giudicelli-Bornard S., Piche T., et al Predictive factors of bleeding related to post-banding ulcer following endoscopic variceal ligation in cirrhotic patients: a case-control study. Alimentary pharmacology \& therapeutics; (2010): 32(2): 225-232.

5. Li P, Zhang ST, Yu ZL, Yu YZ, Ji M, Yu L, Li L, Yan P, Liu YP, Pan JD. Analysis of 
the risk factors in early rebleeding after endoscopic variceal ligation. Zhonghua Xiaohua Neijing Zazhi 2006; 23: 23-26

6. Lo G.H., Chen W.C., Lin C.K., et al Improved survival in patients receiving medical therapy as compared with banding ligation for the prevention of esophageal variceal rebleeding. Hepatology; (2008): 48: 580-587.

7. Xu L., Ji F., Xu Q.W., et al Risk factors for predicting early variceal rebleeding after endoscopic variceal ligation. World journal of gastroenterology: WJG; (2011): 17(28): 3347-3353.

8. Yang M.T., Chen H.S., Lee H.C., et al Risk factors and survival of early bleeding after esophageal variceal ligation. Hepatogastroenterology; (2007): 54(78): $1705-$ 1709.

9. Lee SW, Lee TY, Chang CS Independent factors associated with recurrent bleeding in cirrhotic patients with esophageal variceal hemorrhage. Dig Dis Sci; (2009): 54: 1128-1134.

10. Bosch J. \& Garcia-Pagan J.C. Prevention of variceal re-bleeding. The Lancet ; (2003): 361(9361): 952-954.

11. Fallatah HI, Al Nahdi H, Al Khatabi M Variceal hemorrhage: Saudi tertiary center experience of clinical presentations, complications and mortality. World J Hepatol 2012 September 27; (2012): 4(9): 268-273.

12. Vieira da Rocha EC, D'Amico EA, Caldwell SH et al. A prospective study of conventional and expanded coagulation indices in predicting ulcer bleeding after variceal band ligation. ClinGastroenterol Hepatol; (2009): 7: 988993.

13. Bajaj JS, Schubert CM, Heuman DM et al. Persistence of cognitive impairment after resolution of overt hepatic encephalopathy. Gastroenterology; (2010): 138:2332 2340.

14. Pilete C, Oberty F, Aube C, Rousseelet MC Non-invasive diagnosis of esophageal varices in chronic liver disease. $J$ Hepatol, (1999): 31:867-73.

15. Martins RD, Szejnfeld J, Lima FG, Ferrari AP Endoscopic, Ultrasonographic, US-Doppler parameters as indicators of variceal bleeding in patients with schistomiasis. Dig Dis Sci., (2000): 45:1013-8.
16. Plestina S, Pulaine $R$, Kralik M, Samarzija $M$ Color Doppler ultrasonography is reliable in assessing the risk of esophageal variceal(2005).

17. Said HE, Elsayed EY, Ameen A, Abd Ela $\boldsymbol{H}$ Cytopenia As A Predictor of Oesophageal Varices In Patients With Liver Cirrhosis. Report and Opinion; (2010): 2(7).

18. Thabut D, Ratziu V, Trabut J, Poynard T Prediction of esophageal varices with platelet count/spleen diameter ratio or platelets alone. Gut; (2003): 52:1200-5.

19. Seemakachorn $S$ Mortality and risk factors after endoscopic variceal band ligation.Chiang Mai Medical Bulletin; (2003): 42(2):61-67.

20. Berreta J, Kociak D, Corti $R$ et al. Predictors of intrahospitalary mortality in the upper gastrointestinal variceal bleeding due to chronic liver disease treated endoscopically. Acta Gastroenterol Latinoam; (2008): 38:4350.

21. Blaise M, Pateron D, Trinchet JC et al. Systemic antibiotic therapy prevents bacterial infection in cirrhotic patients with gastrointestinal hemorrhage. Hepatology; (1994): 20:34-38.

22. Moitinho E, Escorsell A, Bandi JC, Salmerón JM, García-Pagán JC, Rodés J, Bosch J Prognostic value of early measurements of portal pressure in acute variceal bleeding. Gastroenterology; (1999): 117: 626-631.

23. Chen W T, Lin C Y, Sheen I S, Huang C $W$, Lin $T$ N, Lin C J, Jeng $W J$, Huang C H, Ho Y P, Chiu C T MELD score can predict early mortality in patients with rebleeding after band ligation for variceal bleeding. World J Gastroenterol; (2011): 17(16): 2120-2125. 